\title{
Competing Through Employee Engagement: A Strategic Perspective of Human Resources Agenda
}

\author{
Rr. Sri Handari Wahyuningsih \\ Ph.D Student Economic and Business Faculty \\ Universitas Brawijaya Malang Indonesia \\ E-mail: ndari_moehdam@yahoo.com \\ Achmad Sudiro \\ Universitas Brawijaya Malang Indonesia \\ EkaAfnan Troena \\ Universitas Brawijaya Malang Indonesia \\ Dodi W Irawanto \\ Universitas Brawijaya Malang Indonesia
}

Received: March 18, 2018

doi:10.5296/jmr.v10i2.12842
Accepted: April 17, $2018 \quad$ Published: April 17, 2018

URL: https://doi.org/10.5296/jmr.v10i2.12842

\begin{abstract}
This is a general review-based study with finding of research is some analysis from previews research. The author conducted a critical review based on empirical study to understand strategic perspective of developing employee engagement from human resources management. The findings showed that Improvement of business competitiveness requires a paradigm of strategic human resource management placing employees as the key factor of success to drive competitiveness. In addressing this challenge, having employee engagement as the key factor of success is strategic human resource agenda. HR managers need to pay more attention about antecedent of employee engagement in order to develop appropriate programs for business competitiveness. Based on previous research studies, this paper provides a conceptual framework that can be used as reference for future research and stimulating programs of HR department for organizational competitiveness building. The
\end{abstract}




\section{Macrothink

most important contribution of this paper is an introduction of new paradigm of strategic human resources agenda to developing programs in attempt of driving organizational competitiveness in unstable environment.

Keywords: Employee Engagement, Strategic Human Resources Agenda 


\section{Introduction}

Resource-based views (Resource Based Value) explain that superior and competitive corporate performance stems from differential capabilities of internal resources of the company (Barney, 2001). This view puts employees in position of a strategic asset in achieving business success and long-term survival. Based on this paradigm, organizations through their Human Resources Department have strategic agenda to build programs of human resource management by paying attention to employee engagement in order to foster business competitiveness in the future.

Organizations face a need to have capability of adaption to uncertainty of external environment that threatens business sustainability. Based on the RBV view, the adaptability to uncertainty can be established when organizations have valuable assets that are difficult to imitate, namely human resources. Therefore, perception of strategies positioning human resources as the key of business success is necessary. With this view, the HR department assumes task of managing sustainably attitudes and behavior of employees that can drive their performance.

The concept of employee engagement has been frequently examined in organizational literature as an employee attitude that is requiring improvement. In his study, Shuck M Brad et al. (2011) concluded the importance of improving employee engagement. Employees with high employee engagement will have ability to adapt to changes that has occurred as the impact of environmental uncertainty.

The concept of engagement begins with the opinion of Kahn (1990) revealing the importance of psychological presence in workplace by distinguishing between engagement and unengagement behavior. This concept is developed from the role theory defined as an experience accompanying a behavioral involvement, involving a releasing physically, cognitively, and emotionally of energy (Kahn, 1990). Psychological presence is described as a condition in which organizational members play their roles deeply in their jobs, expressing their thoughts and feelings, assumptions, and innovating in the workplace. Kahn (1990) suggests four dimensions of psychological presence, namely attention, connection, integration and focus. The psychological presence illustrates the presence of an individual's passion for particular situations. In many organizational literatures, the psychological presence at workplace is manifested as behavior of personal involvement or engagement.

Consideration of the importance of employee engagement as a positive attitude of employees that is necessary to build has been increasing as paying attention to the importance of employee attitudes and behavior. Employee with high engagement is characterized by energy, involvement and role. The engagement contract is expressed as a construct contrary to burnout behavior with its dimensions of fatigue, cynicism, and lack of professional efficacy (Schaufeli et al., 2002). Further study by Schaufeli et al. (2000) concluded that the construct of engagement includes dimensions of vigor, dedication and absorption having a negative correlation with the construct of burnout at workplace.

The paper is allocated into sections. In the first section, author explains purpose and 
methodology of the paper. Second section discusses development of the concept of employee engagement. The third section outlines empirical supports underlying the importance of strategic perspective of employee engagement development by HR managers. Empirical studies are grouped by industry type of some countries. In fourth section, author conducts analysis by positioning employee engagement as the agenda of realizing a work attitude that is driving business competitiveness in global era today.

\section{Purpose, Methodology and Literature Review}

Purpose of the present paper is to provide a comprehensive review literature on employee engagement from various empirical studies with manufacturing and service sector as object of the studies in order to provide an overview for HR managers in scheduling various policies and practices of human resource that can drive business competitiveness through development of positive working attitude that discussed in many literature today, employee engagement. Author made a review literature of various articles from publication journal sources in emerald insight and science direct by using employee engagement or engagement as keyword of the search.

Although the concept of employee engagement has gained much attention and been widely studied empirically, the understanding of definition and measurement of employee engagement are very wide. Kahn (1990) suggested engagement as a form of individual simultaneous expression on a work task bringing a self-relation with the job and others. It is demonstrated by physical, cognitive, and emotional presence, active attitude and showing a result of optimum performance. A person with a high engagement will show condition of "fully connected" to his or her tasks and jobs and, demonstrate full energy in completing tasks, dedicate himself to the job and engage in job activities (Baker, 2011), demonstrate positive attitudes at work, accept organizational values, understanding the business context, have a willingness to work together in order to improve performance for the sake of the organization (Robertson IT. et al., 2010).

Based on the opinion above, HR department needs to create a working climate that can encourage building of employee engagement. In the context of business with full uncertainty, every organization needs employees who have willingness to perform tasks with totality, more self-investing in jobs they have, express themselves physically, cognitively and emotionally, and high levels of energy and enthusiasm in order to promote business performance.

Employee engagement also defines positive behavior of employees as stated by Macey and Schneider (2008) who described the overall meaning of involvement, commitment, passion, enthusiasm and focus on effort and energy. Positive behavior in the workplace is very necessary and it has a strategic aspect in promoting organizational performance. Schaufeli et al. (2002) disclosed three indicators of employee engagement required by organization: 1) Vigor, reflected by condition of employee such as: high energy, endurance, willingness to make hard effort at work, not be tired easily, perseverance in facing adversity; 2) Dedication, describes a strong involvement in the job, enthusiasm, and a sense of self-pride and inspiration; 3) Absorption, reflecting the atmosphere of just like a drowning in the job, a 
feeling that the time is passing quickly and unable to get away from work.

Positive attitude of an employee as expression of high employee engagement includes cognitive and affective attitudes as Simoon (2010) points out in table 2 below.

Table 1. Forms of Employee Engagement

\begin{tabular}{|l|l|l|}
\hline & \multicolumn{1}{|c|}{ Cognitive } & \multicolumn{1}{c|}{ Affective } \\
\hline $\begin{array}{l}\text { Job } \\
\text { engagement }\end{array}$ & $\begin{array}{l}\text { Absorption: } \\
\text { Employees merges } \\
\text { themselves into their } \\
\text { jobs, and they feel } \\
\text { engrossment as they do } \\
\text { the jobs }\end{array}$ & $\begin{array}{l}\text { Energy: } \\
\text { Level of energy } \\
\text { demonstrated by } \\
\text { employees in their jobs. }\end{array}$ \\
\hline $\begin{array}{l}\text { Organizational } \\
\text { engagement }\end{array}$ & $\begin{array}{l}\text { Alignment } \\
\text { Employees' viewpoint to } \\
\text { the organization is in } \\
\text { harmony with } \\
\text { organization's viewpoint } \\
\text { about what to do. }\end{array}$ & $\begin{array}{l}\text { Identification: } \\
\text { Emotional binding } \\
\text { between employees and } \\
\text { organization }\end{array}$ \\
\hline
\end{tabular}

Consistent with explanation above, the importance of employees with high engagement disclosed Rana as the importance of positive behavior at work which is a high expression of engagement that is:

Effort. Employees with high effort will be available to exert their efforts in order to carry out tasks of their job responsibilities. This condition is characterized by high level of energy and mental retention of employees at work, a willingness to spend extra effort while running a job.

Extra-role. Employees will reflect positive behaviors such as availability of completing additional work for the benefit of their organization, showing extra-role behavior, having initiatives to solve problems, offering assistance to co-workers before being asked. These positive behaviors are often referred to in the term "organizational citizenship behavior."

Advocacy is reflected in the "stretch" behavior of activities undertaken in an organization. Employees in such situations will be available to act as a positive advocate for their organization when interacting with people outside of the organization.

The conception and dimensions of employee engagement as explained above give insight to the importance for each organization to pay attention to positive behavior of employees, and of course, this is through programs built by HR department. In the context of behavior, positive or negative response of employees to their jobs is basically influenced by individual perception as a result of psychological experience gained from aspects of work and environment. This condition is shown, among others, by establishment of interpersonal relationships within group, between groups and organizational factors (Kahn, 1990), work experience encouraging attitudes and behavior (Hackman \& Oldham, 1980). If the condition 
is established, employees will have a feeling of fit with the job (job-person fit). This environmental aspect plays a significant role in explaining reasons of employee engagement that can subsequently has an impact on performance.

Organizations that are successfully building an employee engagement will benefit from positive attitudes and individual outcomes and in turn, it will affect organizational performance. Vance R (2006) developed an antecedent and consequent model of employee engagement with the organizational practice as antecedent and the business performance as consequence. In addition, employee performance can be a reciprocal variable. This is supported by McBain Richard (2007) stating that organizational performance is an outcome of the establishment of employee engagement, with indicators such as: 1) Customer service performance, such as improved customer service, customer satisfaction and customer loyalty; 2) management of change, such as establishment of service-oriented organizational culture; 3 ) increased motivation; 4) decreased employee turnover. Thus, it can be understood that employee engagement is not just oriented to the positive attitude of employees, but also a goal of HR strategy in promoting a sustainable performance of organization.

For HR Department, efforts to increase employee engagement can be made by paying attention to the antecedents. Simoon (2010) suggested occupational factor and organizational factor as antecedent of employee engagement, as can be seen in table 2.

Table 2. Antecedent of Employee Engagement

\begin{tabular}{|l|l|}
\hline \multicolumn{1}{|c|}{ Occupational Factor } & \multicolumn{1}{|c|}{ Organizational Factor } \\
\hline 1. Task/job challenge & 1. Wider effect \\
2. Working ethics & 2. Vision \\
3. Level of competition within the & 3. Effective communication \\
4. Interaction Justice \\
organization & 5. Ethics \\
5. Expectation-Career ambition & 6. Effective decision-making \\
6. Factor of individual influence & 7. Customer oriented \\
7. A supporting working & 8. bureaucracy \\
environment & 9. Relationship with employees \\
8. Job setting & 10. Cross-functional collaboration within \\
9. Development & \multicolumn{2}{|c}{ organization } \\
\hline
\end{tabular}

Some other opinions about antecedents of employee engagement have been discussed in some literature, such as opinion of McBain Richard (2007) dividing it into three aspects, namely organizational factors, management and leadership, and quality of working life. Organizational factor includes organizational culture, values and vision, organization/product reputation. Factor of organizational management considers a direct superior leadership, managerial commitment and organizational communication. Factor of working life is reflected by aspects such as a supportive job environment, superior support, job flexibility, employee engagement, work-life balance. A similar opinion is expressed by Rana (2014) distinguishing between job design and job characteristics, relationships with superiors and 
co-workers, work environment, and HR management practices as antecedents of employee engagement.

\section{Empirical Study of Employee Engagement in Various Sectors}

The empirical literature on employee engagement demonstrates attention, urgency and agenda of the importance of improving employee engagement in addressing business developments placing employees as valuable assets and part of business infrastructure. This conceptual paper will review several empirical literatures on similar topic in different organizational contexts of different countries as a basis of synthesizing the urgency for HR managers to pay attention to employee engagement. A short picture of the empirical studies on the manufacturing and service sectors is presented in Table 3, Table 4, and Table 5, respectively.

Table 3. Antecedent and Consequence of Employee Engagement Study in Sector of Manufacturing

\begin{tabular}{|c|c|c|c|c|c|c|}
\hline No & Author & Industry & Object & Country & Antecedent & Consequent \\
\hline 1. & Huang YH, et al., 2016 & Manufacturing & Trucking firms & $\begin{array}{l}\text { United } \\
\text { States of } \\
\text { America }\end{array}$ & $\begin{array}{l}\text { - Employees' safety } \\
\text { climate } \\
\text { - Job satisfaction }\end{array}$ & \\
\hline 2. & $\begin{array}{l}\text { Shuck M.Brad. et al., } \\
2010\end{array}$ & Manufacturing & Employees & $\begin{array}{l}\text { Miami, } \\
\text { Florida }\end{array}$ & $\begin{array}{l}\text { - Environment } \\
\text { - Personal } \\
\text { interaction } \\
\text { - Managerial role } \\
\text { - Personality }\end{array}$ & \\
\hline 3. & McBain R, 2007 & Manufacturing & Employees & $\begin{array}{l}\text { United } \\
\text { States of } \\
\text { America }\end{array}$ & $\begin{array}{l}\text { - Organizational } \\
\text { culture } \\
\text { - Leadership } \\
\text { - Quality of } \\
\text { working life } \\
\text { - Psychological } \\
\text { contract } \\
\text { - Psychological } \\
\text { condition } \\
\text { - Organizational } \\
\text { commitment } \\
\text { - Job satisfaction }\end{array}$ & $\begin{array}{l}\text { - Organizational } \\
\text { Commitment } \\
\text { - Job satisfaction } \\
\text { - Outcome }\end{array}$ \\
\hline 4. & Ajayi OM., et al., 2016 & $\begin{array}{l}\text { Manufacturing } \\
\text { (SME) }\end{array}$ & Employees & Nigeria & $\begin{array}{l}\text { - Organizational } \\
\text { structure } \\
\text { - Knowledge } \\
\text { sharing culture } \\
\text { - Clan culture } \\
\text { - Adhocracy culture }\end{array}$ & \\
\hline
\end{tabular}




\begin{tabular}{|c|c|c|c|c|c|c|}
\hline 5. & $\begin{array}{l}\text { de Sousa and van } \\
\text { Dierendonck. } 2014\end{array}$ & Manufacturing & $\begin{array}{l}\text { Employees of } \\
\text { Companies }\end{array}$ & Portuguese & $\begin{array}{l}\text { - Servant leadership } \\
\text { - Psychological } \\
\text { Empowerment } \\
\text { - Post-Merger } \\
\text { Organization }\end{array}$ & \\
\hline 6. & Lara & Manufacturing & Managers & Spain & $\begin{array}{l}\text { - } \text { Managerial } \\
\text { competences } \\
\text { - OLC } \\
\text { (organizational } \\
\text { Learning } \\
\text { Capability) }\end{array}$ & \\
\hline 7. & $\begin{array}{l}\text { GhorbannejadParisa } \\
\text { and Esakhani Ahmad, } \\
2016\end{array}$ & $\begin{array}{l}\text { Manufacturing } \\
\text { (Automotive) }\end{array}$ & Employees & Iran & Individual Traits & $\begin{array}{l}\text { - Job Satisfaction } \\
\text { - Commitment } \\
\text { - OCB } \\
\text { - Performance }\end{array}$ \\
\hline 8. & $\begin{array}{l}\text { Albrecht L Simon, } \\
2012\end{array}$ & Manufacturing & $\begin{array}{l}\text { Multi-national } \\
\text { mining } \\
\text { companies }\end{array}$ & Australia & $\begin{array}{l}\text { - Job resources } \\
\text { - Organizational } \\
\text { Resources } \\
\text { - Team Resources }\end{array}$ & $\begin{array}{l}\text { - Commitment } \\
\text { - Performance }\end{array}$ \\
\hline
\end{tabular}

Empirical studies of antecedent and consequences of employee engagement with objects of service sector are presented in table 4 as follows.

Table 4. Empirical Studies of Antecedent and consequence of Employee Engagement Studies in Service Sector

\begin{tabular}{|c|c|c|c|c|c|c|}
\hline No & Author & Industry & Object & Country & Antecedent & Consequence \\
\hline 1. & $\begin{array}{l}\text { Park J and } \\
\text { Gursoy } \text { D, } \\
2012\end{array}$ & Hospitality Service & $\begin{array}{l}\text { Middle-Top } \\
\text { Managers }\end{array}$ & America & & - Turnover intention \\
\hline 2. & $\begin{array}{l}\text { Karatepe OM } \\
\text { et al., }\end{array}$ & Hospitality Service & $\begin{array}{l}\text { Employees of } \\
\text { Five Stars } \\
\text { Hotel }\end{array}$ & Turk & Challenging stressor & $\begin{array}{l}\text { - Affective } \\
\text { commitment } \\
\text { - Job Performance }\end{array}$ \\
\hline 3. & Karatepe 2013 & Hospitality Service & $\begin{array}{l}\text { Frontline } \\
\text { Employees of } \\
\text { Hotels }\end{array}$ & Iran & $\begin{array}{l}\text { Politics } \\
\text { Organization }\end{array}$ & $\begin{array}{l}\text { - Extra Role } \\
\text { Performance } \\
\text { - affective } \\
\text { Commitment } \\
\text { - ToI }\end{array}$ \\
\hline 4. & $\begin{array}{l}\text { Paek et al., } \\
2015\end{array}$ & Hospitality Service & Employees & $\begin{array}{l}\text { South } \\
\text { Korea }\end{array}$ & $\begin{array}{l}\text { Psychological } \\
\text { capital }\end{array}$ & $\begin{array}{l}\text { - JS } \\
\text { - Affective } \\
\text { Commitment }\end{array}$ \\
\hline 5. & $\begin{array}{l}\text { Jung HS and } \\
\text { Yoon, } 2015\end{array}$ & Hospitality Service & Employees & Korea & Pay Satisfaction & \\
\hline 6. & Zhang et al., & Service & Hotel & China & Identification & \\
\hline
\end{tabular}




\begin{tabular}{|c|c|c|c|c|c|c|}
\hline No & Author & Industry & Object & Country & Antecedent & Consequence \\
\hline & 2017 & & $\begin{array}{l}\text { HRDs in } \\
\text { China }\end{array}$ & & $\begin{array}{l}\text { Organization } \\
\text { Respect } \\
\text { Pride }\end{array}$ & \\
\hline 7. & $\begin{array}{l}\text { Nazirowais } \\
\text { and Islam } \\
\text { UlJamid, } 2017\end{array}$ & Education service & $\begin{array}{l}600 \\
\text { respondents of } \\
\text { Higher } \\
\text { Education }\end{array}$ & Delhi, India & POS & $\begin{array}{l}\text { - affective } \\
\text { Commitment } \\
\text { - Employees' } \\
\text { performances }\end{array}$ \\
\hline 8. & Saks, 2006 & Education service & $\begin{array}{l}\text { Participants } \\
\text { (employees) } \\
\text { of courses }\end{array}$ & Canada & $\begin{array}{l}\text { - Job characteristics } \\
\text { - Perception of } \\
\text { organizational } \\
\text { support } \\
\text { - Perception of } \\
\text { managerial } \\
\text { support } \\
\text { - reward and } \\
\text { recognition, } \\
\text { Procedural justice, } \\
\text { and distributive } \\
\text { justice }\end{array}$ & $\begin{array}{l}\text { - Job satisfaction } \\
\text { - Commitment } \\
\text { - Turn-over } \\
\text { intention } \\
\text { - OCB }\end{array}$ \\
\hline 9. & $\begin{array}{l}\text { Wefald\& } \\
\text { Downey. } \\
2009 .\end{array}$ & Education Service & $\begin{array}{l}\text { Students of } \\
\text { secondary and } \\
\text { high schools }\end{array}$ & $\begin{array}{l}\text { Kansas } \\
\text { (U.S.A), } \\
\text { UK }\end{array}$ & & Job satisfaction \\
\hline 10. & $\begin{array}{l}\text { Schaufeli WB, } \\
\text { et al., } 2002\end{array}$ & Education Service & $\begin{array}{l}\text { University } \\
\text { students }\end{array}$ & Spain & Burnout & \\
\hline 11. & $\begin{array}{l}\text { Al Tit AA et } \\
\text { al., } 2015\end{array}$ & Education Service & Employees & Jordan & $\begin{array}{l}\text { Relationship of } \\
\text { employees } \\
\text { Trust } \\
\text { Communication } \\
\text { Justice Organization } \\
\text { Organizational and } \\
\text { superior supports } \\
\text { Motivation and } \\
\text { Feedback } \\
\text { reward system } \\
\text { Career }\end{array}$ & Job satisfaction \\
\hline 12. & $\begin{array}{l}\text { Ben Suzanne et } \\
\text { al., } 2015\end{array}$ & Education Service & $\begin{array}{l}\text { Lecturers and } \\
\text { Engineers }\end{array}$ & Australia & $\begin{array}{l}\text { Participation } \\
\text { Involvement }\end{array}$ & $\begin{array}{l}\text { - Job satisfaction } \\
\text { - Organizational } \\
\text { performance }\end{array}$ \\
\hline 13. & $\begin{array}{l}\text { Giallonardo } \\
\text { ML, } 2010\end{array}$ & Education Service & $\begin{array}{l}\text { Nursing } \\
\text { Graduates, } 63\end{array}$ & America & $\begin{array}{l}\text { Leadership } \\
\text { Authentic }\end{array}$ & Job satisfaction \\
\hline 14. & $\begin{array}{l}\text { Ogbuanya and } \\
\text { Chukwuedo, }\end{array}$ & Education Service & Lecturers & Nigeria & $\begin{array}{l}\text { Commitment } \\
\text { Job Crafting }\end{array}$ & $\begin{array}{l}\text { Job satisfaction } \\
\text { Commitment }\end{array}$ \\
\hline
\end{tabular}




\section{Macrothink}

Journal of Management Research

ISSN 1941-899X

2018, Vol. 10, No. 2

\begin{tabular}{|c|c|c|c|c|c|c|}
\hline No & Author & Industry & Object & Country & Antecedent & Consequence \\
\hline & 2017 & & & & & \\
\hline 15. & $\begin{array}{l}\text { Suhartanto et } \\
\text { al., } 2017\end{array}$ & Retail Service & Employees & $\begin{array}{l}\text { Bandung, } \\
\text { Indonesia }\end{array}$ & & Job Satisfaction \\
\hline 16. & $\begin{array}{l}\text { Zhang, Avery, } \\
\text { Bergsteiner, } \\
\text { More. } 2014\end{array}$ & Retail Service & Employees & $\begin{array}{l}\text { Sydney, } \\
\text { Australia }\end{array}$ & Leadership & $\begin{array}{l}\text { Employees } \\
\text { Characteristics } \\
\text { need for } \\
\text { achievement, } \\
\text { sensitivity justice, } \\
\text { and need for clarity }\end{array}$ \\
\hline 17. & $\begin{array}{l}\text { Schaufeli WB } \\
\text { and Baker AB, } \\
2004\end{array}$ & $\begin{array}{l}\text { Financial services } \\
\text { (insurance, } \\
\text { Occupational } \\
\text { Safety, pension } \\
\text { fund) and Home } \\
\text { Care }\end{array}$ & Employees & Netherland & - Job resources & Turn over intention \\
\hline 18. & $\begin{array}{l}\text { Rahman Ur } \\
\text { Ubaid et al., } \\
2017\end{array}$ & $\begin{array}{l}\text { Insurance and } \\
\text { financial services }\end{array}$ & Employees & Pakistan & $\begin{array}{l}\text { Psychological } \\
\text { Contract }\end{array}$ & $\begin{array}{l}\text { Employees } \\
\text { performances } \\
\text { Jobs satisfaction }\end{array}$ \\
\hline 19. & $\begin{array}{l}\text { Ugwu } \quad \text { O } \\
\text { Fabian, } 2014\end{array}$ & $\begin{array}{l}\text { Financial services } \\
\text { and Pharmacy }\end{array}$ & $\begin{array}{l}\text { Bank, } \\
\text { Pharmacy }\end{array}$ & Nigeria & $\begin{array}{l}\text { Trust } \\
\text { empowerment }\end{array}$ & \\
\hline 20. & $\begin{array}{l}\text { Hakanan JJ. Et } \\
\text { al., } 2008\end{array}$ & Health services & $\begin{array}{l}\text { Health } \\
\text { workers }\end{array}$ & Finland & $\begin{array}{l}\text { - Home resources } \\
\text { - Home demand } \\
\text { - Job resources }\end{array}$ & \\
\hline 21. & $\begin{array}{l}\text { Xu Jessica and } \\
\text { Thomas } \\
\text { Helena, } 2011\end{array}$ & Insurance service & $\begin{array}{l}\text { Insurances' } \\
\text { employees }\end{array}$ & $\begin{array}{l}\text { New } \\
\text { Zealand }\end{array}$ & $\begin{array}{l}\text { Leadership } \\
\text { Tenure }\end{array}$ & \\
\hline 22. & $\begin{array}{l}\text { Nada Al } \\
\text { Mehrzi NA } \\
\text { and Singh SK, } \\
2016\end{array}$ & $\begin{array}{l}\text { Governmental } \\
\text { service }\end{array}$ & Employees & $\begin{array}{l}\text { United } \\
\text { Arab } \\
\text { Emirates }\end{array}$ & $\begin{array}{l}\text { Culture } \\
\text { Leadership } \\
\text { Team work } \\
\text { POS }\end{array}$ & Motivation \\
\hline 23. & $\begin{array}{l}\text { Gupta Manish., } \\
\text { et al., } 2016\end{array}$ & $\begin{array}{l}\text { Governmental } \\
\text { service }\end{array}$ & $\begin{array}{l}\text { Public } \\
\text { servants, } \\
\mathrm{n}=293\end{array}$ & India & $\begin{array}{l}\text { Psychological } \\
\text { Capital }\end{array}$ & OCB I and OCB O \\
\hline 24. & $\begin{array}{l}\text { OgeErcan, et } \\
\text { al., } 2017\end{array}$ & $\begin{array}{l}\text { Air transportation } \\
\text { service }\end{array}$ & Employees & Istanbul & $\begin{array}{l}\text { Leadership } \\
\text { Paternalistic }\end{array}$ & $\begin{array}{l}\text { WFC } \\
\text { Workplace loneliness }\end{array}$ \\
\hline
\end{tabular}

Studies on employee engagement using the manufacturing firms as objects (table 3 ) linked the employee engagement to individual outcomes such as organizational commitment, job satisfaction, organizational citizenship behavior, and performance. Some of these studies concluded that a mutual relationship was found between employee engagement and satisfaction, and between employee engagement and organizational commitment. A study of Huang YH et al., (2016) concluded that employee engagement is influenced by job 
satisfaction. Meanwhile, based on study of Ghorbannejad Parisa and Esakhani Ahmad (2016), the job satisfaction is an impact of employee engagement. In contrast, findings of McBain Richard's (2007) study proved a mutual relationship between employee engagement and satisfaction, and between employee engagement and commitment.

Based on an empirical study with the object of service organization, Jung HS and Yoon (2015) explicitly concluded that salary satisfaction is an antecedent of employee engagement (table 4). However, Saks (2006) concluded in his study that job satisfaction is an impact of employee engagement. Based on these studies, red lines can be drawn that employee engagement affects individual outcomes such as organizational commitment, job satisfaction, organizational citizenship behavior, and performance. Relationship between employee engagement and job satisfaction can be mutual in nature.

Based on empirical studies with various industries in one study period (table 5) as object, provide a similar picture of antecedents and consequent of employee engagement. The cross-organizational study shows various aspects of employee engagement antecedents that are necessary paid attention, namely job, organizational (processes) and individual aspects. Assessment of employee about the job aspect and overall organizational process will determine how high the employee's attachment to the organization. This will affect effectiveness of the job implementation, as described in table 5 as follows.

Table 5. Empiric Studies of Antecedent and consequence of Employee Engagement in Various Sectors of Organizations

\begin{tabular}{|c|c|c|c|c|c|c|}
\hline No & Author & Group & Object & Country & Antecedent & Consequent \\
\hline 1. & $\begin{array}{l}\text { YasinGhadi, } \\
\text { Fernando, } \\
\text { Caputi. } 2013\end{array}$ & $\begin{array}{l}\text { Various } \\
\text { industries }\end{array}$ & Employees & $\begin{array}{l}\text { Sydney, } \\
\text { Australia }\end{array}$ & $\begin{array}{l}\text { - } \text { Transformational } \\
\text { leadership } \\
\text { - Employees' } \\
\text { perceptions of } \\
\text { meaning } \\
\text { in work positively } \\
\text { - } \quad \text { Perceptions of } \\
\text { meaning in work }\end{array}$ & \\
\hline 2. & $\begin{array}{l}\text { Avery } \\
\& \text { Bergsteiner. } \\
2014\end{array}$ & $\begin{array}{l}\text { Various } \\
\text { industries }\end{array}$ & Employees & $\begin{array}{l}\text { Sydney, } \\
\text { Australia }\end{array}$ & $\begin{array}{ll}\text { - } & \text { Paradigm of visionary } \\
\text { - } & \text { and organic } \\
\text { nal-stylesemployee } \\
\text { engagement (-). } \\
\text { - } \quad \text { Behavioral-outcome }\end{array}$ & \\
\hline 3. & $\begin{array}{l}\text { Karanika-Murray } \\
\text { et al., } 2015\end{array}$ & $\begin{array}{l}\text { Various } \\
\text { industries }\end{array}$ & $\begin{array}{l}\text { Professional, } \\
\text { administrative,s } \\
\text { enior executive }\end{array}$ & America & $\begin{array}{l}\text { Organizational } \\
\text { identification }\end{array}$ & Job satisfaction \\
\hline 4. & $\begin{array}{l}\text { PopliSavna and } \\
\text { Rizvi A Irvan, } \\
2015\end{array}$ & $\begin{array}{l}\text { Banking } \\
\text { service } \\
\text { telecommunicat }\end{array}$ & Managers & India & $\begin{array}{l}\text { Service orientation } \\
\text { Transformational } \\
\text { leadership }\end{array}$ & \\
\hline
\end{tabular}




\begin{tabular}{|l|l|l|l|l|l|l|}
\hline & $\begin{array}{l}\text { ion, Education, } \\
\text { Research }\end{array}$ & & & & \\
\hline
\end{tabular}

\section{Synthesis: Strategic Agenda of the Department of Human Resources}

A business phenomenon guiding the importance of adaptive ability for every business entity needs to put the aspect of human resources as human capital as a competitiveness resource into its agenda. Various studies summarized in this paper prove the importance of every sector of an organization to drive its efforts in developing human resource programs in attempts of encouraging positive employee attitudes indicated by employee engagement, thereby promoting performance improvements and other individual outcomes, which in turn, it will produce positive results on organizational performance and competitiveness. The HR program demonstrates efforts of an organization to create a job environment, working environment and management system encouraging quality of work life.

Inability of the organization to create a working environment will affect positive perception of employees so that it can reduce their engagement in their jobs. It is in line with Khan's analysis (1992) suggesting that organizational factors (Hochschild, 1983) can increase or weaken motivation and sense of meaningful of employees in workplace. When such condition occurs within an organization, the engagement can also decrease further.

It is important to note that employee engagement can be built by every organization through various human resource programs supported by good organizational process. In attempt of innovation and gaining competitive advantage, an organization need to have employees who are willing to exert extra energy and work in totality, but it should not necessary create workaholic employees. Concept of engagement is different from work aholicism and organizational commitment (Bakker et al., 2008). Organizations need to pay attention to antecedents of employee engagement sourced from job resources and personal resources (Bakker et al., 2008). Job resources include autonomy, supervisory, coaching and performance feedback. While personal resources include employee optimism, self-efficacy, and self-esteem.

Similar opinion suggested by Simon (2010) that antecedents of employee engagement, namely characteristics of a working environment factors cover job role and wider range of other organizational features describing the job as a whole. The working environment will shape experiences of employees in their jobs, and it can encourage employees to have engagements or otherwise, encourage them to turn away from job responsibilities.

Human resources department plays a central role in building a working attitude and outcome. Therefore, they need to have a strategic paradigm in developing various HR policies encouraging employee engagement that ultimately resulting in positive aspects of individual and organizational outcomes. Positive consequence at workplace was also expressed by some experts, among the others: individual outcome such as work performance and satisfaction (Bakker, 2008) and, organizational performance such as profitability, revenue growth, earnings per share and employee turnover (Harter et al., 2002). Employees with high engagement and commitment to organization will encourage the competitive advantage of the 
company. Employees will have high productivity and no intention to leave the organization.

\section{Conclusion}

The present study is a critical literature review study of previous research studies related to employee engagement with various studies of antecedents and consequences. Relationshipbetween factors that shape employee engagement and its impact on individuals and organizations provides an explanation of the importance of employee management agenda. Based on the discussion above, the following conceptual framework is prepared:

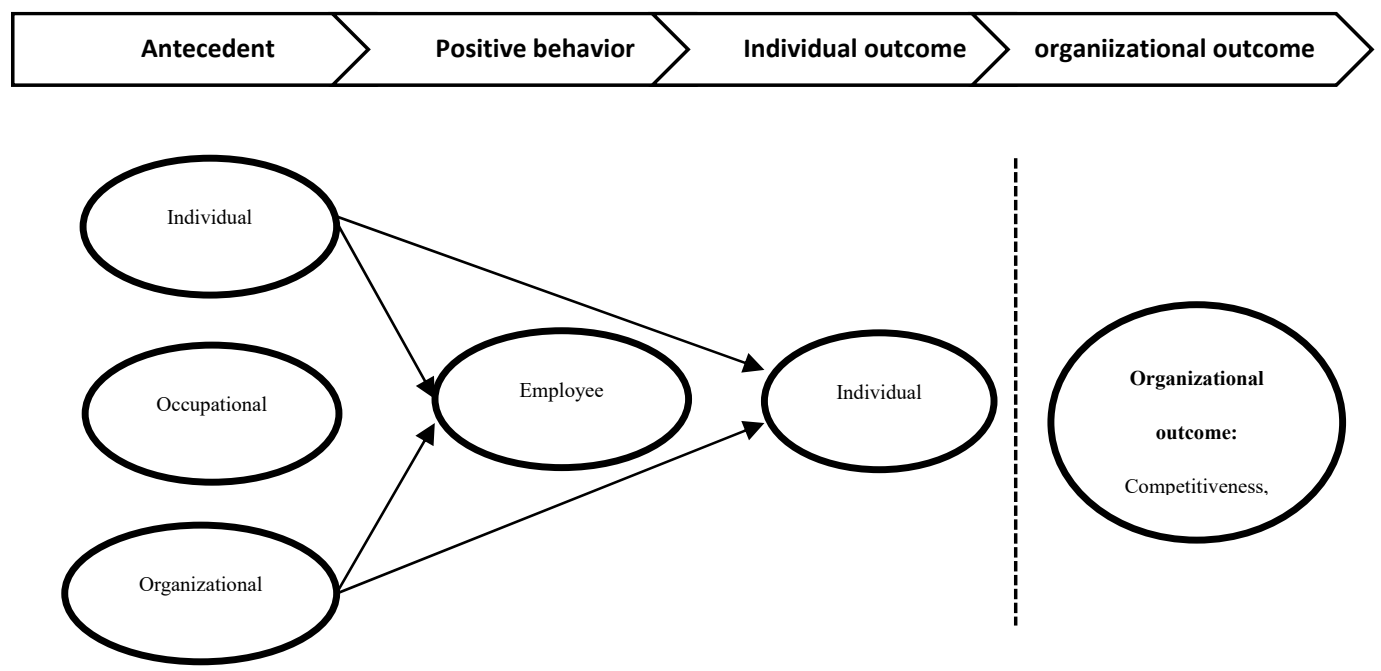

Figure 1. Antecedents and consequences of employee engagement

Every organization has a responsibility of maintaining long-term effectiveness so that it is necessary to put human resource as the main asset of the organization through a strategic program of human resource management that is capable to encourage employee engagement. Aspects that are necessary to consider include individual aspects, occupational aspects and organizational aspects. With the employee engagement, then individual outcome such as satisfaction, commitment, performance, and organizational citizenship behavior can be improved and consequently, it will provide a positive impact on organizational outcomes.

\section{References}

Ajayi, O. M., Odusanya, K., \& Morton, S. (2017). Stimulating employee ambidexterity and employee engagement in SMEs. Management Decision, 55(4), 662-680. https://doi.org/10.1108/MD-02-2016-0107

AL-TIT, A., \& Hunitie, M. (2015). The Mediating Effect of Employee Engagement between Its Antecedents and Consequences. Journal of Management Research, 7(5), 47. https://doi.org/10.5296/jmr.v7i5.8048

Bakker, A. B. et al. (2008). Work engagement: An emerging concept in occupational health psychology. Work and Stress, 22(3), 187-200. https://doi.org/10.1080/02678370802393649

Bakker, A. B. (2011). An Evidence-Based Model of Work Engagement. Current Directions in Psychological Science, 20(4), 265-269. https://doi.org/10.1177/0963721411414534 
Barney, J. B. (2001). Resource-based theories of competitive advantage: A ten-year retrospective on the resource-based view. Journal of Management, 27(6), 643-650. https://doi.org/10.1016/S0149-2063(01)00115-5

Benn, S., Teo, S. T. T., \& Martin, A. (2015). Employee participation and engagement in working for the environment. Personnel Review, 44(4), 492-510. https://doi.org/10.1108/PR-10-2013-0179

Brad Shuck, M., Rocco, T. S., \& Albornoz, C. A. (2011). Exploring employee engagement from the employee perspective: implications for HRD. Journal of European Industrial Training, 35(4), 300-325. https://doi.org/10.1108/03090591111128306

Burke, R. J. et al. (2009). Work engagement among hotel managers in Beijing, China: potential antecedents and consequences. Tourism Review, 64(3), 4-18. https://doi.org/10.1108/16605370910988791

Cattermole, G., Johnson, J., \& Jackson, D. (2014). Employee engagement creates a brighter economic future at Jupiter Hotels. Strategic HR Review, 13(2), 81-85. https://doi.org/10.1108/SHR-11-2013-0110

Chaudhary, R. (2017). Corporate social responsibility and employee engagement: can CSR help in redressing the engagement gap?. Social Responsibility Journal, 13(2), 323-338. https://doi.org/10.1108/SRJ-07-2016-0115

Clara C. Romero, K., \& L. Lamadrid, R. (2014). Rethinking corporate social responsibility within the sustainability agenda. Journal of Global Responsibility, 5(2), 180-202. https://doi.org/10.1108/JGR-06-2014-0023

Costa, P. L., Passos, A. M., \& Bakker, A. B. (2016). The work engagement grid: predicting engagement from two core dimensions. Journal of Managerial Psychology, 31(4), 774-789. https://doi.org/10.1108/JMP-11-2014-0336

Fearon, C., McLaughlin, H., \& Morris, L. (2013). Conceptualising work engagement. European Journal of Training and Development, 37(3), 244-256. https://doi.org/10.1108/03090591311312723

Ferreira, P., \& Real de Oliveira, E. (2014). Does corporate social responsibility impact on employee engagement?. Journal of Workplace Learning, 26(3/4), 232-247. https://doi.org/10.1108/JWL-09-2013-0070

Ghorbannejad, P., \& Esakhani, A. (2016). Capacity to engage: studying role of individual differences in work engagement - evidences from Iran. Journal of Management Development, 35(9), 1174-1183. https://doi.org/10.1108/JMD-02-2015-0029

Giallonardo, L. M., Wong, C. A., \& Iwasiw, C. L. (2010). Authentic leadership of preceptors: Predictor of new graduate nurses' work engagement and job satisfaction. Journal of Nursing Management, 18(8), 993-1003. https://doi.org/10.1111/j.1365-2834.2010.01126.x

Gill, R. (2015). Why the PR strategy of storytelling improves employee engagement and adds 
value to CSR: An integrated literature review. Public Relations Review, 41(5), 662-674. Elsevier Inc. https://doi.org/10.1016/j.pubrev.2014.02.012

Gruman, J. A., \& Saks, A. M. (2011). Performance management and employee engagement. Human Resource Management Review, 21(2), 123-136. Elsevier Inc. https://doi.org/10.1016/j.hrmr.2010.09.004

Gupta, M., Shaheen, M., \& Reddy, P. K. (2017). Impact of psychological capital on organizational citizenship behavior. Journal of Management Development, 36(7), 973-983. https://doi.org/10.1108/JMD-06-2016-0084

Hakanen, J. J., Schaufeli, W. B., \&Ahola, K. (2008). The job demands-resources model: A three-year cross-lagged study of burnout, depression, commitment, and work engagement. Work and Stress, 22(3), 224-241. https://doi.org/10.1080/02678370802379432

Hallberg, U. E., \& Schaufeli, W. B. (2006). "Same same" but different? Can work engagement be discriminated from job involvement and organizational commitment? European Psychologist, 11(2), 119-127. https://doi.org/10.1027/1016-9040.11.2.119

Harter, J. K., Schmidt, F. L., \& Hayes, T. L. (2002). Business-unit-level relationship between employee satisfaction, employee engagement, and business outcomes: A meta-analysis. Journal of Applied Psychology, 87(2), 268-279. https://doi.org/10.1037/0021-9010.87.2.268

Hassan, A., \& Ahmed, F. (2011). Authentic leadership, trust and work engagement. World Academy of Science Engineering Technology, 80, 750-756. Available at: http://ejournal.narotama.ac.id/files/v6-3-24.pdf.

Huang, Y.-H. et al. (2016). Beyond safety outcomes: An investigation of the impact of safety climate on job satisfaction, employee engagement and turnover using social exchange theory as the theoretical framework. Applied Ergonomics, 55, 248-257. Elsevier Ltd. https://doi.org/10.1016/j.apergo.2015.10.007

J., A. (2014). Determinants of employee engagement and their impact on employee performance. International Journal of Productivity and Performance Management, 63(3), 308-323. https://doi.org/10.1108/IJPPM-01-2013-0008

Jung, H. S., \& Yoon, H. H. (2016). What does work meaning to hospitality employees? The effects of meaningful work on employees' organizational commitment: The mediating role of job engagement. International Journal of Hospitality Management, 53, 59-68. Elsevier Ltd. https://doi.org/10.1016/j.ijhm.2015.12.004

Kahn, W. A. (1990). Psychological Conditions of Personal Engagement and Disengagement At Work. Academy of Management Journal, 33(4), 692-724. https://doi.org/10.2307/256287

Karanika-Murray, M. et al. (2015). Organizational identification, work engagement, and job satisfaction. Journal of Managerial Psychology, 30(8), 1019-1033. https://doi.org/10.1108/JMP-11-2013-0359

Karatepe, O. M. (2013). Perceptions of organizational politics and hotel employee outcomes. 
International Journal of Contemporary Hospitality Management, 25(1), 82-104. https://doi.org/10.1108/09596111311290237

Karatepe, O. M. et al. (2014). Does work engagement mediate the effects of challenge stressors on job outcomes? Evidence from the hotel industry. International Journal of Hospitality Management, 36, 14-22. Elsevier Ltd. https://doi.org/10.1016/j.ijhm.2013.08.003

Keating, L. A., \& Heslin, P. A. (2015). The potential role of mindsets in unleashing employee engagement. Human Resource Management Review, 25(4), 329-341. Elsevier Inc. https://doi.org/10.1016/j.hrmr.2015.01.008

Lu, L. et al. (2016). Work engagement, job satisfaction, and turnover intentions. International Journal of Contemporary Hospitality Management, 28(4), 737-761. https://doi.org/10.1108/IJCHM-07-2014-0360

Macey, W. H., \& Schneider, B. (2008). The Meaning of Employee Engagement. Industrial and Organizational Psychology, 1(1), 3-30. https://doi.org/10.1111/j.1754-9434.2007.0002.x

Mackay, M. M., Allen, J. A., \& Landis, R. S. (2017). Investigating the incremental validity of employee engagement in the prediction of employee effectiveness: A meta-analytic path analysis. Human Resource Management Review, 27(1), 108-120. Elsevier B.V. https://doi.org/10.1016/j.hrmr.2016.03.002

Mayo, A. (2016). The measurement of engagement. Strategic HR Review, 15(2), 83-89. https://doi.org/10.1108/SHR-02-2016-0012

McDermott, C. C. (2009). Corporate Agenda 21: a unified global approach to CSR and sustainability. Corporate Communications: An International Journal, 14(3), 286-302. https://doi.org/10.1108/13563280910980078

Al Mehrzi, N., \& Singh, S. K. (2016). Competing through employee engagement: a proposed framework. International Journal of Productivity and Performance Management, 65(6), 831-843. https://doi.org/10.1108/IJPPM-02-2016-0037

Nazir, O., \& Islam, J. U. (2017). Enhancing organizational commitment and employee performance through employee engagement. South Asian Journal of Business Studies, 6(1), 98-114. https://doi.org/10.1108/SAJBS-04-2016-0036

Öge, E., Çetin, M., \& Top, S. (2018). The effects of paternalistic leadership on workplace loneliness, work family conflict and work engagement among air traffic controllers in Turkey. Journal of Air Transport Management, 66(February 2017), 25-35. https://doi.org/10.1016/j.jairtraman.2017.10.003

Paek, S. et al. (2015). Why is hospitality employees' psychological capital important? The effects of psychological capital on work engagement and employee morale. International Journal of Hospitality Management, 50, 9-26. Elsevier Ltd. https://doi.org/10.1016/j.ijhm.2015.07.001

Park, J., \& Gursoy, D. (2012). Generation effects on work engagement among U.S. hotel 
employees. International Journal of Hospitality Management, 31(4), 1195-1202. Elsevier Ltd. https://doi.org/10.1016/j.ijhm.2012.02.007

Popli, S., \& Rizvi, I. A. (2015). Exploring the relationship between service orientation, employee engagement and perceived leadership style: a study of managers in the private service sector organizations in India. Journal of Services Marketing, 29(1), 59-70. https://doi.org/10.1108/JSM-06-2013-0151

Rahman, U. U. et al. (2017). Does team orientation matter? Linking work engagement and relational psychological contract with performance. Journal of Management Development, 36(9), 1102-1113. https://doi.org/10.1108/JMD-10-2016-0204

Rahn, D. L. et al. (2016). Journal of Organizational Effectiveness : People and Performance Article information. Journal of Organizational Effectiveness: People and Performance, 3(1), 43-69. https://doi.org/10.1108/JOEPP-04-2015-0016

Rama Devi, V. (2009). Employee engagement is a two - way street. Human Resource Management International Digest, 17(2), 3-4. https://doi.org/10.1108/09670730910940186

Robertson, I. T., \& Cooper, C. L. (2010). Full engagement: the integration of employee engagement and psychological well - being. Leadership \& Organization Development Journal, 31(4), 324-336. https://doi.org/10.1108/01437731011043348

Schaufeli, W. B. et al. (2002). The Measurement of Engagement and Burnout: a Two Sample Confirmatory Factor Analytic Approach. Journal of Happiness Studies, 3, 71-92. https://doi.org/10.1023/A:1015630930326

Schaufeli, W. B., \& Bakker, A. B. (2004). Job demands, job resources, and their relationship with burnout and engagement: A multi-sample study. Journal of Organizational Behavior, 25(3), 293-315. https://doi.org/10.1002/job.248

Singhapakdi, A. et al. (2015). The impact of incongruity between an organization's CSR orientation and its employees' CSR orientation on employees' quality of work life. Journal of Business Research, 1sevier B.V., 68(1), 60-66. https://doi.org/10.1016/j.jbusres.2014.05.007

Taneja, S., Sewell, S. S., \& Odom, R. Y. (2015). A culture of employee engagement: a strategic perspective for global managers. Journal of Business Strategy, 36(3), 46-56. https://doi.org/10.1108/JBS-06-2014-0062

Teck Hui, L. (2008). Combining faith and CSR: a paradigm of corporate sustainability. International Journal of Social Economics, 35(6), 449-465. https://doi.org/10.1108/03068290810873429

Tziner, A., \& Tanami, M. (2014). Journal of Work and Organizational Psychology. Journal of Work and Organizational Psychology. Colegio Oficial de Psicólogos de Madrid, 30(2), 67-74. https://doi.org/10.1016/j.rpto.2017.09.003

Ugwu, F. O., Onyishi, I. E., \& Rodríguez-Sánchez, A. M. (2014). Linking organizational trust with employee engagement: the role of psychological empowerment. Personnel Review, 
43(3), 377-400. https://doi.org/10.1108/PR-11-2012-0198

Vance, R. J. (2004). Engagement and Commitment Engagement and. SHRM Foundation, $1-53$.

Zhang, T. et al. (2014a). Do follower characteristics moderate leadership and employee engagement?. Journal of Global Responsibility, 5(2), 269-288. https://doi.org/10.1108/JGR-04-2014-0016

Zhang, T. et al. (2014b). The relationship between leadership paradigms and employee engagement. Journal of Global Responsibility, 5(1), 4-21. https://doi.org/10.1108/JGR-02-2014-0006 